\title{
Multiple organ involvement and ICU considerations for the care of acute liver failure (ALF) and acute on chronic liver failure (ACLF) in children
}

\author{
Leslie A. Mataya ${ }^{1}$, Vijay Srinivasan ${ }^{1,2}$, Elizabeth B. Rand ${ }^{3,4}$, Alicia M. Alcamo ${ }^{1,2} \wedge$ \\ ${ }^{1}$ Division of Critical Care Medicine, Children's Hospital of Philadelphia, Philadelphia, PA, USA; ${ }^{2}$ Department of Anesthesiology and Critical Care \\ Medicine, University of Pennsylvania, Philadelphia, PA, USA; ${ }^{3}$ Division of Gastroenterology, Hepatology, and Nutrition, Children's Hospital of \\ Philadelphia, Philadelphia, PA, USA; ${ }^{4}$ Department of Pediatrics, University of Pennsylvania, Philadelphia, PA, USA \\ Contributions: (I) Conception and design: LA Mataya, AM Alcamo; (II) Administrative support: None; (III) Provision of study materials or patients: \\ None; (IV) Collection and assembly of data: None; (V) Data analysis and interpretation: None; (VI) Manuscript writing: All authors; (VII) Final \\ approval of manuscript: All authors. \\ Correspondence to: Alicia M. Alcamo, MD. Division of Critical Care Medicine, 3401 Civic Center Boulevard, Philadelphia, PA 19104, USA. \\ Email: alcamoa@email.chop.edu.
}

\begin{abstract}
Liver disease results in approximately 15,000 pediatric hospitalizations per year in the United States and is a significant burden to child health. Major etiologies of liver failure and indications for transplantation in children include: biliary atresia, metabolic/genetic conditions, toxins, infections, tumors, and immune-mediated liver/biliary injury. Children requiring transplantation are placed on the United Network of Organ Sharing waitlist including those with acute liver failure (ALF) and acute on chronic liver failure (ACLF). ALF is a clinical syndrome in which a previously healthy child develops rapid-onset hepatic dysfunction, and becomes critically ill with multiple organ dysfunction within days. ACLF, by contrast, is generally described as an acute decompensation of pre-existing chronic liver disease (CLD) brought on by a precipitating event, with higher risk of mortality. Children with ALF and ACLF receive multidisciplinary care in pediatric intensive care units (ICUs) due to multiple organ system involvement and high risk of decompensation in these patients. The care of these patients requires a holistic approach that addresses the complex interplay between hepatic and extra-hepatic organ systems. This review will define and describe ALF and ACLF in the pediatric population, and outline the effects of ALF and ACLF on individual organ systems with diagnostic and management considerations in the ICU while awaiting liver transplantation.
\end{abstract}

Keywords: Acute liver failure (ALF); acute on chronic liver failure (ACLF); organ system; critical care; pediatrics

Submitted Nov 10, 2020. Accepted for publication Feb 23, 2021.

doi: $10.21037 / \mathrm{tp}-20-375$

View this article at: http://dx.doi.org/10.21037/tp-20-375

\section{Introduction}

Although the true prevalence of liver failure in children is not known, it is estimated that approximately 15,000 children are hospitalized for liver disease each year in the United States (1,2). Many of these children are cared for in pediatric intensive care units (ICUs), due to multiple organ system involvement and high risk of life-threatening decompensation in these patients. Major etiologies of liver failure and indications for transplantation in children include: biliary atresia, metabolic/genetic conditions, toxins, infections, cirrhosis, tumors, and immune-mediated liver/

$\wedge$ ORCID: 0000-0002-5406-5681. 
Table 1 OPTN criteria for pediatric Status 1A and Status 1B for liver transplantation (4)

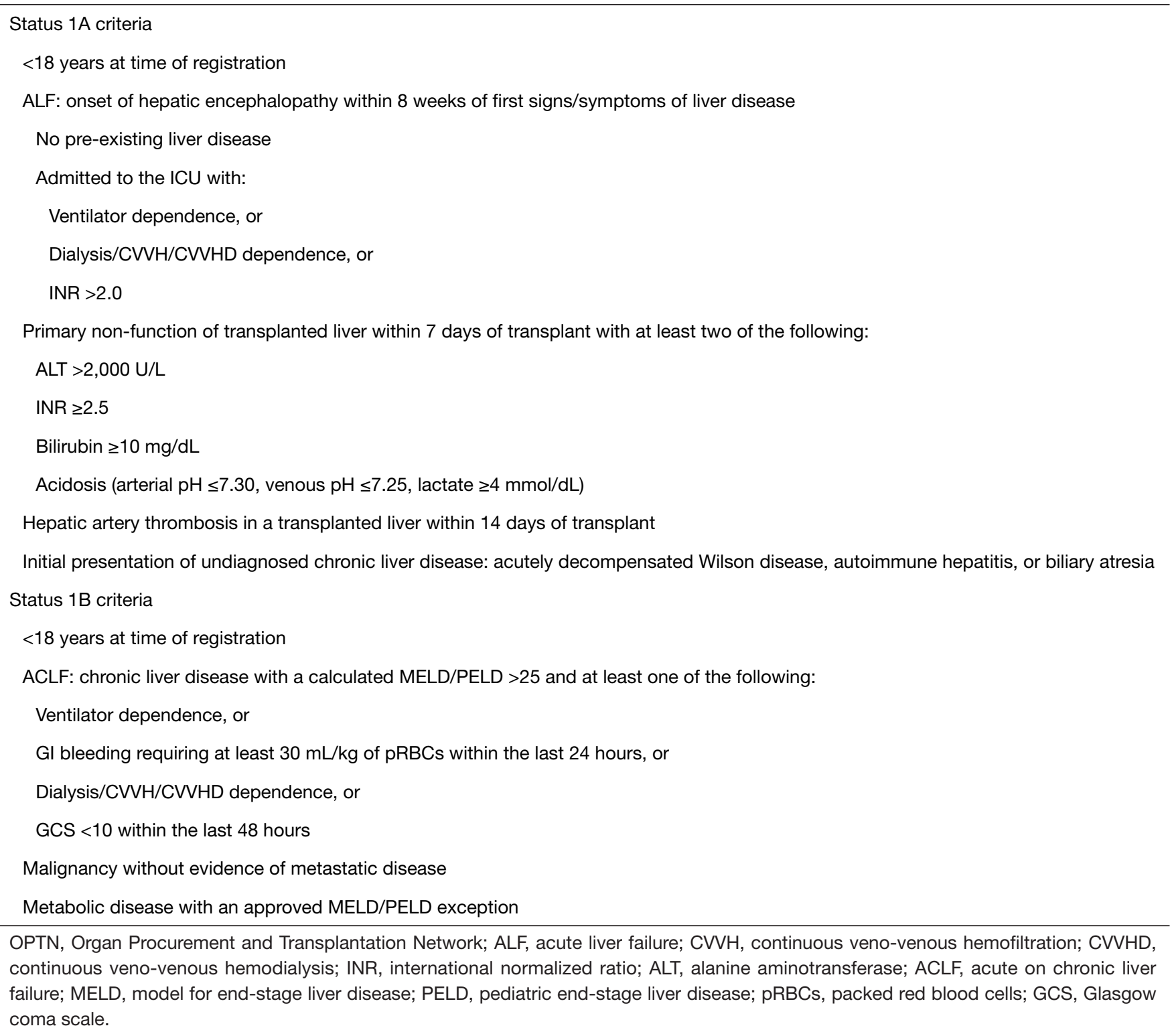

biliary injury (3).

Children who require liver transplantation are placed on the United Network for Organ Sharing (UNOS) waitlist, prioritized by acuity. Children with acute liver failure (ALF) are listed as Status 1A (highest priority), and those with acute on chronic liver failure (ACLF) are listed as Status 1B (next highest in priority) (Table 1) (4). This review article will define both ALF and ACLF, discuss the multiple organ system involvement in ALF and ACLF, and examine specific ICU considerations in the care of these patients while awaiting liver transplantation.

\section{$A L F$}

ALF is well-described in children, with defined criteria to identify these patients, and sufficient data are available on the natural course of ALF, both with and without transplantation (5). ALF is a clinical syndrome in which a previously healthy child develops rapid-onset hepatic dysfunction, and becomes critically ill with multiple organ dysfunction within days. The pediatric ALF (PALF) study group defines ALF as biochemical evidence of acute liver injury shorter than 8 weeks in duration with either (I) 
Table 2 Treatments specific to acute liver failure etiologies

\begin{tabular}{ll}
\hline Reason for acute liver failure & Treatment \\
\hline Acetaminophen overdose & N-acetylcysteine \\
Herpes simplex virus & Acyclovir \\
Autoimmune hepatitis & Corticosteroids \\
Wilson disease & Low copper diet, copper chelators (D-penicillamine, trientine) \\
Inborn errors of metabolism & Varies based on the diagnosis \\
Hemophagocytic lymphohistiocytosis & Etoposide, dexamethasone \\
Gestational alloimmune liver disease & Exchange transfusions, high-dose intravenous immunoglobin \\
\hline
\end{tabular}

coagulopathy with INR (international normalized ratio) $\geq 1.5$ with hepatic encephalopathy (HE) or (II) INR $\geq 2$ without $\mathrm{HE}$ and not corrected by intravenous vitamin $\mathrm{K}$ (5). Given the relatively low rate of spontaneous recovery without transplantation $(15-20 \%)$ and the potential for rapid deterioration, ALF is a medical emergency and requires immediate transfer to a pediatric liver transplant center for management $(6,7)$.

No diagnosis is made in $54 \%$ of children with ALF despite comprehensive evaluation (5). In those children (birth to 18 years of age) with ALF who receive a diagnosis, herpes simplex virus (HSV) is the most common infectious etiology, and acetaminophen overdose is the most common drug-induced etiology. In infants, metabolic disease is a common etiology, while autoimmune hepatitis is a common etiology among older children (8). Etiologies also vary by geographic location: for example, viral hepatidities are more common in developing countries (9). The etiology of ALF is an important predictor of outcome: some diagnoses have specific treatments that can dramatically affect the clinical course of ALF and potentially obviate the need for liver transplantation or, in some cases, these treatments are required for continued disease management for other organs even after liver transplantation (Table 2) $(10,11)$.

\section{$A C L F$}

In contrast to ALF, there are no well-established criteria for ACLF in pediatrics. In adults, several definitions specify criteria for the diagnosis of ACLF (12-14). All of these definitions generally describe ACLF as an acute decompensation of pre-existing chronic liver disease (CLD) brought on by a precipitating event, with higher risk of mortality. While individual definitions of decompensation of liver function in adults with ACLF vary, similarities include elevated levels of serum bilirubin and INR, and presence of ascites or HE. In addition, ACLF in adults is accompanied by extra-hepatic organ system failures, and the severity of ACLF can be graded based upon the number of hepatic and extra-hepatic organ systems affected. However, none of these definitions are validated for use in children. In spite of this limitation, many pediatric studies have used these adult criteria to identify ACLF (15-18). In an attempt to recognize these children and prioritize them more urgently for transplantation, the Organ Procurement and Transplantation Network (OPTN) created criteria for Status 1B (see Table 1) (4). Children with certain metabolic diseases and malignancies can also qualify for Status 1B, but do not meet the definition of ACLF.

Biliary atresia, autoimmune hepatitis, and Wilson disease are the most common underlying CLD diagnoses identified in pediatric ACLF. Common precipitating events in ACLF appear to vary by region: the hepatotropic viruses are common in studies from Southeast Asia, while sepsis and gastrointestinal (GI) bleeding are common in studies from Europe. The mortality rate for pediatric ACLF in all of these studies ranged between $20-30 \%$, and was five times higher in ACLF compared to CLD (15-18).

\section{Multiple organ system involvement and associated ICU management of ALF and ACLF}

The hallmark of both ALF and ACLF is involvement of extra-hepatic organ systems, sometimes resulting in organ dysfunction and failure. The following sections outline the effects of ALF and ACLF on individual organ systems with diagnostic and management considerations in the ICU while awaiting liver transplantation. In addition to management of specific organ system derangements in ALF and ACLF, consideration should also be given to 
Table 3 Hepatic encephalopathy classifications and management

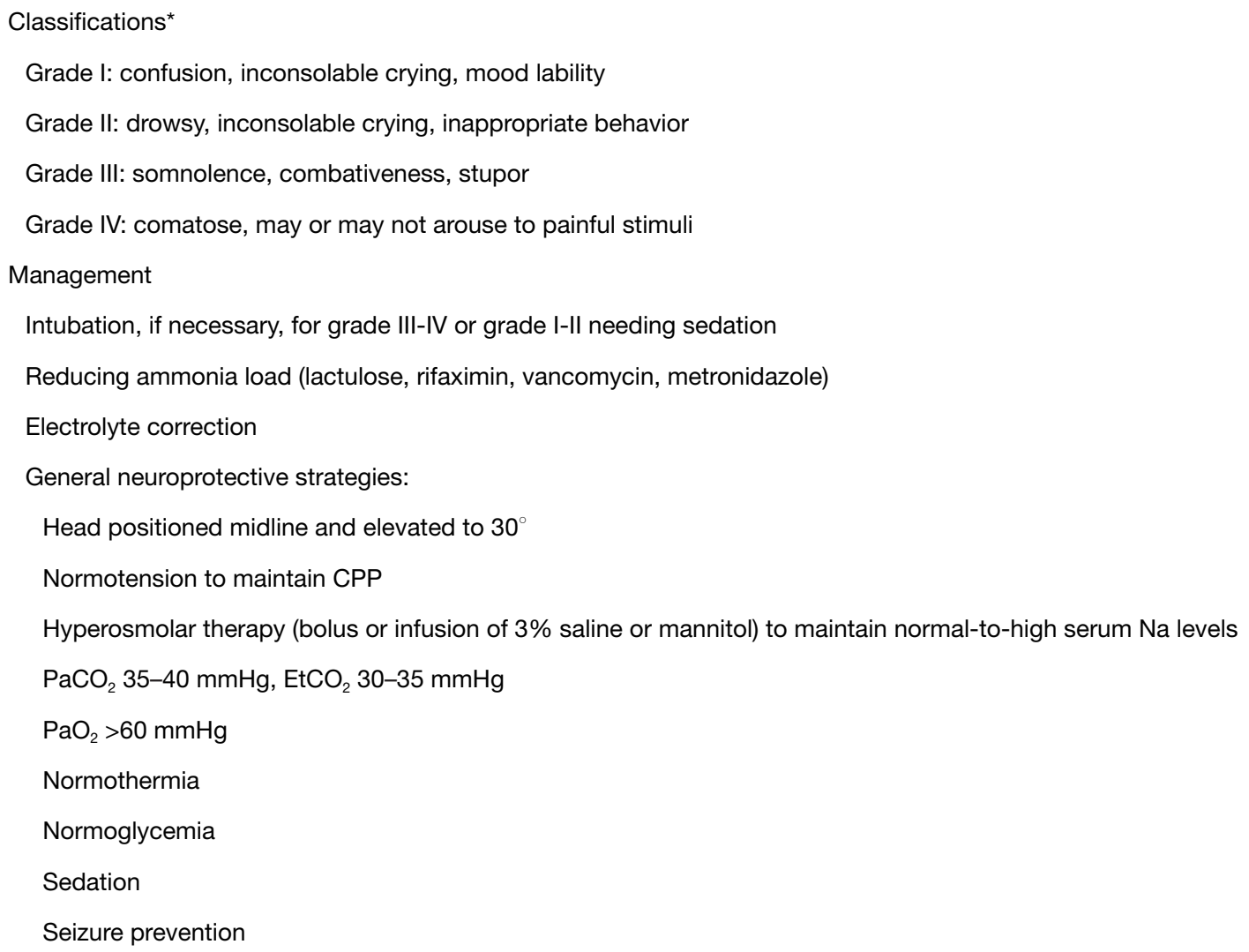

*, the grading of hepatic encephalopathy is dependent on age-appropriate responses. CPP, cerebral perfusion pressure; Na, sodium; $\mathrm{PaCO}_{2}$, arterial carbon dioxide; $\mathrm{EtCO}_{2}$, end tidal carbon dioxide; $\mathrm{PaO}_{2}$, arterial oxygen.

initiating specific therapies for underlying liver disease for management while awaiting liver transplantation (Table 2).

\section{Respiratory}

Respiratory derangements are common in children with ALF and ACLF. Respiratory alkalosis may occur due to central stimulation of respiration due to reduced hepatic clearance and accumulation of short-chain fatty acids in the cerebrospinal fluid (19). Children with ALF or ACLF may develop acute hypoxemic respiratory failure and require endotracheal intubation for a myriad of reasons, including sepsis, volume overload, pulmonary hemorrhage, or acute respiratory distress syndrome (ARDS). Pulmonary hemorrhage, in particular, is considered a terminal stage of liver failure, and is associated with high mortality both pre- and post-transplant (20). In addition to management of overt acute respiratory failure, endotracheal intubation should be considered in children with grade III or IV HE who require airway protection due to neurologic failure (Table 3). At lower stages of encephalopathy (grades I or II), the use of sedation to control agitation should be avoided as use of these medications can lead to an inadequate respiratory drive with resulting hypercapnia leading to cerebral vasodilation which may cause an acute increase in intracranial pressure (ICP). Another important reason to avoid sedation is to preserve the ability to follow serial clinical exams to monitor progression of encephalopathy.

An uncommon yet clinically significant complication of CLD is hepatopulmonary syndrome (HPS), which affects up to $20 \%$ of children with CLD and can be seen in ACLF, pre- or post-transplant (21). HPS is defined as impaired oxygenation due to nitric oxide-mediated vasodilation of the pulmonary vasculature, in the setting of CLD. This leads to hypoxemia through three mechanisms:

(I) Ventilation-perfusion (VQ) mismatch resulting from normal ventilation in the "over-perfused" parts of the lung; 
(II) Vascular shunting, leading to rapid exit of blood from the pulmonary vasculature before it is adequately oxygenated;

(III) Impaired diffusion of oxygen through thickened capillary walls.

HPS is diagnosed by agitated saline contrast echocardiography or lung perfusion scan. Children with CLD and HPS who develop ACLF are at higher risk for hypoxemic respiratory failure. Treatments that target inhibition of nitric oxide (such as methylene blue) or promote vasoconstriction (such as indomethacin) can be attempted, however, neither of these has shown significant clinical benefit in humans. Liver transplantation is the only definitive treatment for HPS $(21,22)$. Interestingly, there have been case reports in the pediatric population that show inhaled nitric oxide leading to a paradoxical improvement in HPS-related hypoxemia and respiratory failure in the immediate post-transplant period (23-25).

Another pulmonary complication of CLD, and therefore seen in ACLF, is porto-pulmonary hypertension, which is the development of pulmonary arterial hypertension $(>25 \mathrm{mmHg}$ ) in the setting of liver disease or portal hypertension. It is mediated by increased circulating endothelin-1 and decreased prostacyclin synthase. While evidence of pulmonary hypertension can be seen on echocardiogram, a cardiac catheterization is required to confirm the diagnosis. Treatment strategies include prostacyclin analogues (such as treprostinil), endothelin receptor antagonists (such as bosentan), and phosphodiesterase inhibitors (such as sildenafil). Although liver transplantation is a definitive treatment for mild and moderate porto-pulmonary hypertension, severe portopulmonary hypertension (which is rare in children) remains a contraindication to transplantation due to high associated mortality and poor prognosis (22). Patients with severe porto-pulmonary hypertension may require combined liver and lung or heart transplantation. There is at least one case report of pediatric patient who underwent veno-venous extracorporeal membrane oxygenation (VV-ECMO) as a rescue for severe porto-pulmonary hypertension after liver transplantation, however this effort was unsuccessful (26).

Hepatic hydrothorax is a known complication of liver cirrhosis and is a manifestation of in ACLF in adults, but is rare in pediatric patients $(27,28)$. Hepatic hydrothorax occurs in the absence of cardiopulmonary disease and is typically associated with significant ascites in CLD. In cirrhosis, pleural effusions may occur secondary to the hypoalbuminemic state that results in decreased oncotic pressure and therefore fluid accumulation in the pleural cavity (29). Management of a hepatic hydrothorax is typically conservative and primarily focuses on management of the associated ascites and portal hypertension, which is discussed later in this the review.

If endotracheal intubation is necessary in a patient with ALF or ACLF, it should be performed by the most experienced provider due to concomitant coagulopathy and swollen airway structures that could precipitate bleeding with multiple attempts. A lung protective ventilatory strategy is recommended, with tidal volumes of $6-8 \mathrm{~mL} / \mathrm{kg}$ and peak inspiratory pressures (PIPs) $<30 \mathrm{cmH}_{2} \mathrm{O}$ to maintain pulse oximetry oxygen saturation $\left(\mathrm{SpO}_{2}\right)>96 \%$ and arterial carbon dioxide $\left(\mathrm{PaCO}_{2}\right)$ levels of $35-40 \mathrm{mmHg}$. If possible, avoid high positive end expiratory pressure (PEEP) that could increase ICP and hepatic venous pressure by providing positive intrathoracic pressure and decreasing venous return to the heart. If the patient has ARDS, the traditional consensus guidance of permissive hypercapnia and hypoxemia is not indicated in patients with ALF or ACLF who may also have concomitant increased ICP. Regular chest physiotherapy is not contraindicated, however, this should be done with caution given higher risk of bleeding from associated coagulopathy (30).

\section{Cardiovascular}

When shock is present in ALF and ACLF, it is typically distributive in nature, either from the liver failure itself, or due to acquired sepsis. The presence of inflammatory cytokines leads to a hyperdynamic circulatory failure state with elevated levels of catecholamines. However, this state is characterized by low mean arterial blood pressure (MAP) due to peripheral vasodilation from elevated nitric oxide levels resulting in lowered systolic and diastolic blood pressures. Additionally, there are numerous derangements of the microcirculation due to shunting and occlusion with tissue hypoxia and elevated serum lactate levels. Once it is determined that adequate fluids have been given to expand intravascular volume, therapy with inotropes should be considered. Norepinephrine is the preferred firstline inotrope in liver failure as it counteracts peripheral vasodilation, increasing both systolic and diastolic blood pressure. Vasopressin may be considered as a second-line agent to treat hypotension refractory to norepinephrine. Dopamine is not recommended as it can have deleterious effects on peripheral vasodilation. There may also be a component of relative adrenal insufficiency in these 
patients, so stress dose steroids should be considered in patients with fluid- and vasoactive-resistant shock (31).

$\mathrm{N}$-acetylcysteine (NAC) was evaluated as a therapeutic agent in ALF to support cardiovascular hemodynamics and oxygen transport for both ALF related to acetaminophen and ALF not associated with acetaminophen (32). Some adult and pediatric studies suggest potential benefits including longer transplant-free survival, particularly for patients with lower grades of HE $(33,34)$. However, a prospective pediatric study evaluated the use of NAC compared to placebo in pediatric ALF not related to acetaminophen toxicity. This study found no improvement in long-term survival as well as lower rates of 1-year transplant-free survival, especially in patients less than 2 years old (35). Therefore, the use of NAC for cardiovascular support in liver failure to improve transplantfree survival has no clear benefit.

CLD can lead to cirrhotic cardiomyopathy, which is characterized by systolic and diastolic dysfunction in the setting of cirrhosis, and in the absence of known cardiac disease. The underlying pathophysiology is likely secondary to increased myocardial wall stiffness caused by hypertrophy, fibrosis, and sub-endothelial edema, all of which lead to high filling pressures in the left atrium and ventricle. Usually these changes are mild and reversible after liver transplantation, and the pharmacological management of cirrhotic cardiomyopathy includes beta blockers, diuretics, and angiotensin-converting enzyme (ACE) inhibitors. If patients with CLD develop ACLF, those patients with underlying cirrhotic cardiomyopathy are at higher risk for cardiac dysfunction in the setting of critical illness $(36,37)$.

\section{Neurologic}

$\mathrm{HE}$ is a neuropsychiatric syndrome seen in CLD, ALF and ACLF, in the absence of pre-existing brain disease. $\mathrm{HE}$ is difficult to assess in children, and is associated with increased morbidity, including moderate to severe neurologic deficits in survivors (including poor school/ work performance, and altered executive functioning). $\mathrm{HE}$ is also associated with increased mortality, affecting up to $55 \%$ in ALF children with grade III-IV (literature unclear in ACLF) (38). Symptoms can range from mild confusion to stupor and coma. The pathophysiology of $\mathrm{HE}$ is multifactorial in nature, involving the production of glutamine from ammonia, which leads to increased intracellular osmolarity in astrocytes in the brain, resulting in cerebral edema. Glutamine also has a direct toxic effect on neurons. Increased cerebral blood flow from vasodilation may result from glutamate release and trigger of neuronal nitric oxide synthetase (n-NOS) to increase nitric oxide production through an increase in $\mathrm{N}$-methyl-D-aspartate (NMDA) activation (39). In addition, inflammatory cytokines such as interleukin (IL)-1 $\beta$, IL-6, and tumor necrosis factor (TNF)- $\alpha$ cause endovascular inflammation that can disturb cerebral blood flow (40).

While HE develops rapidly in ALF, its onset can be rather gradual and subtle in those children with CLD, becoming more evident when they develop ACLF due to sudden hepatic decompensation from a precipitating event (dehydration, electrolyte imbalance, infection, or bleeding). Symptomatic management of HE includes endotracheal intubation if necessary for airway protection in severely altered sensorium (grade III-IV encephalopathy), reducing the ammonia load with medications such as lactulose or rifaximin, gradual electrolyte correction, IV antibiotics, neuroprotective measures (Table 3), and, ultimately, liver transplantation $(8,30)$.

Increased ICP, defined as $>20 \mathrm{mmHg}$, is a direct complication of cerebral edema associated with ALF and, less commonly, ACLF $(41,42)$. Cerebral edema is usually diagnosed clinically by identifying hypertension, bradycardia, irregular respirations, altered mental status, or an abnormal neurologic exam. Intubation may be necessary to protect the airway and to allow for sedation that will prevent spikes in ICP. However, the benefits of intubation must be weighed with the risk of being unable to serially monitor the neurologic exam in a sedated, intubated patient. Invasive ICP monitoring is not routinely recommended in the setting of profound coagulopathy, in the absence of definitive data to support better clinical outcomes.

Strategies for decreasing ICP include: head positioned in the midline and elevated to $30^{\circ}$, normotension to maintain cerebral perfusion pressure (CPP), titration of serum sodium levels to $145-150 \mathrm{mmol} / \mathrm{L}$ (using $3 \%$ hypertonic saline), and maintaining arterial carbon dioxide $\left(\mathrm{PaCO}_{2}\right)$ levels of $35-40 \mathrm{mmHg}$. Of note, hypernatremia goals may not be feasible in ACLF due to the baseline hyponatremia. Serum sodium goals require careful consideration in patients with ACLF if aggressive ICP control is needed due to risk of worsening ascites and fluid overload. Other neuroprotective strategies include: normothermia, maintaining arterial oxygen $\left(\mathrm{PaO}_{2}\right)$ levels $>60 \mathrm{mmHg}$, and normoglycemia. Monitoring for the presence of seizures and initiation of prompt treatment are integrals part of neuroprotection to decrease the metabolic demand of the brain tissue and prevent 
propagation of injury. Ammonia-scavenging therapies, such as benzoate, phenylacetate, and phenylbutyrate, are neuroprotective by providing alternative pathways for nitrogen disposal and therefore reducing hyperammonemia in ALF and ACLF (30).

Patients with liver failure and HE who require endotracheal intubation should be kept comfortable to avoid acute increases in ICP. Use of opioids for sedation is recommended and utilization of sedation protocols with targeted depth of sedation are helpful for optimal comfort. Short-acting opioid infusions such as remifentanil may facilitate brief interruptions to better follow neurological exams in these patients. In general, benzodiazepines are avoided due to alterations of metabolism of this drug class in liver failure that result in accumulation of active metabolites with prolonged drug effects. The prolonged drug effects can serve to confound the neurologic exam in the setting of HE. Clustering nursing care is also beneficial to minimize stimulation and ICP elevations (8).

\section{Hematologic}

Anemia, thrombocytopenia and leukopenia are common in ACLF. The pathophysiology associated with these cytopenias is multifactorial, including splenic sequestration secondary to portal hypertension, alterations in bone marrow stimulating factors including thrombopoietin and erythropoietin, bone marrow suppression, consumptive coagulopathy, and potential for ongoing blood loss (43). Management of these cytopenias includes prevention of GI bleeding, including the use of GI prophylaxis, and measures to reduce portal hypertension. If bleeding is present, appropriate resuscitation should be undertaken in the ICU, with close attention to fluid status given the risk of fluid overload. In addition to leukopenia seen in ACLF, both ALF and ACLF are pro-inflammatory states, with monocyte and macrophage dysfunction central to their disease progression. This leads to hepatic inflammation and concurrent systemic immunosuppression. Infections are common and can result in life-threatening complications in both ALF and ACLF, requiring a high index of suspicion for diagnosis as the presentation can often be subtle (44).

Apart from von Willebrand factor (vWF) which is synthesized by the endothelium, all other coagulation factors are synthesized in the liver. Therefore, coagulopathy in ALF and ACLF is largely a reflection of synthetic liver dysfunction. Admission to the pediatric ICU is indicated in any child with liver disease who has a measured INR level $>4$. As levels of both pro-coagulant and anticoagulant proteins are decreased, these patients rarely experience spontaneous bleeding. The index of suspicion should be high for disseminated intravascular coagulation (DIC) which can occur concomitantly with ALF and ACLF, particularly with superimposed sepsis. Measurement of factor VIII/ vWF levels can help make this distinction between DIC due to sepsis (low factor VIII/vWF levels) and coagulopathy due to worsening liver disease (normal to high factor VIII/wVF levels). Coagulopathy may also lead to GI bleeding, as well as neurovascular events, both ischemic and hemorrhagic in nature $(45,46)$.

Coagulopathy should not be preemptively corrected unless the patient is actively bleeding, or there are invasive procedures planned (intubation, central line insertion, endoscopy, lumbar puncture, etc.). In the absence of indications, aggressive correction of coagulopathy is not recommended as this can obscure the INR trend. INR levels are an important consideration in establishing listing for liver transplantation and also serve as a prognostic indicator in both ALF and ACLF. It is recommended that vitamin $\mathrm{K}$ be empirically given to all patients with ALF and ACLF. Fresh frozen plasma (FFP) contains all coagulation factors, inhibitors of coagulation, and fibrinolytic factors. However, due to risk of volume overload, infection, and transfusion-associated liver injury, FFP therapy should be used judiciously. Cryoprecipitate therapy may be administered when fibrinogen level $<100 \mathrm{mg} / \mathrm{dL}$. If therapy with FFP, cryoprecipitate and platelets fails to deter bleeding, desmopressin (to release $\mathrm{vWF}$ and factor VIII), factor VIIa concentrate, and prothrombin complex concentrate may be considered $(47,48)$. These products with their lower volumes are especially useful in the setting of fluid overload to reverse the coagulopathy. Tranexamic acid and aminocaproic acid may be beneficial in patients with alterations of the fibrinolytic pathways (49-51). Platelet levels should be maintained $>10,000-20,000$, (or $>50,000$ for any invasive procedures). Red blood cell transfusions should also be used judiciously to target hemoglobin levels of $8-9 \mathrm{~g} / \mathrm{dL}$.

\section{Renal}

Acute kidney injury (AKI) is common in ALF and ACLF. Etiologies of AKI include acute tubular necrosis, hypovolemia, sepsis, nephrotoxic medications, and functional renal failure [including hepatorenal syndrome (HRS)]. The prevalence of AKI in ALF is estimated 
to be as high as $17.5 \%$ and is associated with increased mortality (8). The prevalence of AKI in ACLF is unknown in the pediatric population. Serum creatinine values are affected by muscle mass and liver synthetic function, both of which are impaired in liver disease, making serum creatinine a less sensitive biomarker for identifying AKI in these children. A trend of a patient's creatinine levels should be used to determine if there is concerns for kidney injury $(8,52)$.

HRS is seen only in CLD, and is a type of AKI secondary to renal vasoconstriction and intravascular fluid depletion that occurs in CLD. Several theories exist to explain the etiology of HRS, including portal hypertension with bacterial translocation, an exaggerated inflammatory response, decreased renal blood flow due to impaired autoregulation, intra-abdominal hypertension, and cardiac dysfunction. Presence of HRS can predispose a patient with CLD to severe renal failure if they progress to ACLF (52).

The true prevalence of HRS in pediatrics is unknown, though it is thought to be quite rare. HRS is subdivided into HRS type 1 and HRS type 2. HRS type 1 develops acutely, is often severe, and typically associated with ACLF as it is brought upon by a precipitating event. In contrast, HRS type 2 is a slow deterioration in renal function that is directly proportional to the deterioration in liver function. Studies in adults with CLD show that the presence of either type of HRS increases mortality. Liver transplantation can be a definitive treatment, however, simultaneous liver-kidney transplantation may be indicated if the HRS is longstanding and kidney function is not thought to be recoverable (52).

Early management of AKI should focus on reducing ongoing kidney injury by minimizing exposure to IV contrast agents and nephrotoxic drugs, avoiding overdiuresis, restoring intravascular volume and maintaining renal perfusion pressure. Accepted indications for initiating renal replacement therapy in ALF or ACLF include: variceal bleeding, severe metabolic acidosis, hyperammonemia, hyper-/hyponatremia, hyperkalemia, severe fluid overload, and uremia. Continuous veno-venous hemofiltration is preferable over intermittent hemodialysis, as there are less hemodynamic shifts with the former and can be better tolerated by the patient. Anticoagulation with either heparin or calcium citrate is acceptable, depending on center experience and the underlying degree of coagulopathy (53-55).

\section{GI}

Ascites is a common complication of CLD, and its occurrence can predict decompensation into ACLF. Common risk factors for development of ascites include: low serum albumin, GI bleeding, and recent therapeutic endoscopy. Sodium and fluid restriction may be helpful in the prevention of ascites, and further medical management includes diuresis (typically with an aldosterone antagonist and a thiazide diuretic) and $25 \%$ albumin infusion to increase intravascular oncotic pressure. Therapeutic paracentesis is indicated for refractory ascites that is associated with intra-abdominal hypertension, respiratory distress, or AKI (30).

Portal hypertension is a common GI complication of CLD, and a known precipitating risk factor for ACLF in patients with CLD. Portal hypertension is clinically characterized by impaired or reversal of portal blood flow, development of collaterals (including esophageal varices), splenomegaly, and resulting cytopenias. Varices are abnormal venous communications between the portal and systemic circulations that are formed to decompress the portal venous system. Varices typically develop in the lower esophagus and stomach, and can result in acute large volume bleeding. Other complications of portal hypertension include splenomegaly, thrombocytopenia, ascites, bacterial translocation due to intestinal permeability, growth failure, and poorer quality of life $(56,57)$.

Portal hypertension is the most common cause of severe acute GI bleeding in children, and can often be the presenting feature of previously unrecognized CLD. Primary prevention of acute GI bleeding in a patient with portal hypertension may include acid blockade. Nonselective beta blockers are not routinely recommended as first line therapy for prophylaxis against variceal bleeding due to pediatric patients' reliance on tachycardia to compensate for hypovolemic and hemorrhagic shock (57). Some centers proactively perform endoscopy for ligation or sclerotherapy. Transjugular portosystemic shunt (TIPS) may be considered in a patient with refractory variceal bleeding or ascites due to portal hypertension (56).

When patients with portal hypertension present with acute bleeding (manifesting as hematemesis, melena, or hematochezia), they can quickly develop hypovolemic and hemorrhagic shock. Airway, breathing, and circulation should be assessed appropriately. Large bore intravenous catheters should be inserted and resuscitation with crystalloid and blood products should promptly commence. It is important to avoid over-resuscitation or administering fluids too rapidly, as this can exacerbate bleeding by further increasing the pressure in the portal system. Invasive blood 
Table 4 Clinical and ascitic fluid criteria for spontaneous bacterial peritonitis

Fever
Abdominal pain/tenderness
Diarrhea
Ascitic fluid analysis:
Gram stain/culture
Polymorphonuclear leukocyte count $\geq 250$ cells $/ \mathrm{mm}^{3}$

pressure monitoring is recommended to titrate resuscitation to normalization of heart rate and blood pressure. Gastric decompression is used in order to quantify blood loss as well as remove blood from the stomach that could precipitate encephalopathy due to high protein load. Additional treatments including antibiotics, acid blockade, vitamin $\mathrm{K}$, sucralfate, and octreotide should be administered. Octreotide is a synthetic somatostatin analog that decreases portal pressure. Octreotide is administered as a bolus dose $(1-2 \mathrm{mcg} / \mathrm{kg})$ and followed by a continuous infusion of $1-2 \mathrm{mcg} / \mathrm{kg} / \mathrm{hour}$ and is generally well tolerated but may cause transient hypertension, bradycardia and hyperglycemia (58). Endoscopy is indicated for all children presenting with variceal bleeds, unless there is hemodynamic instability or other contraindication. Endoscopic interventions include variceal ligation or sclerotherapy. Balloon tamponade is very effective in temporarily controlling bleeding, but should only be used in an intubated and sedated child as a bridge to definitive treatment or transfer to a liver transplantation center (56).

\section{Infectious diseases}

Children with ALF or ACLF are at very high risk for infection and may exhibit potential signs including temperature $<36$ or $>38{ }^{\circ} \mathrm{C}$, derangements in white blood cell count $(\mathrm{WBC})<4 \mathrm{k}$ or $>12 \mathrm{k} / \mathrm{mm}^{3}$, tachycardia, hypotension, etc. Patients with portal hypertension and associated splenomegaly typically have baseline leukopenia, and a change from their baseline WBC could be a sign of infection requiring evaluation. Common types of infection in these patients are spontaneous bacterial peritonitis (SBP), pneumonia, urinary tract infection, and bacteremia, typically caused by gram positive cocci or gram-negative bacilli (Staphylococcus, Streptococcus, Enterococcus, Escherichia coli, Pseudomonas, Klebsiella, etc.). Up to $23 \%$ of bacterial isolates from children with ALF and ACLF have been found to be multidrug resistant (59). Empiric broad-spectrum antibiotic therapy should be commenced immediately upon suspicion of infection in these patients. Broad-spectrum bacterial coverage with either piperacillin-tazobactam or a thirdgeneration cephalosporin is preferred. Vancomycin should be considered in patients with prior history of methicillinresistant Staphylococcus aureus (MRSA) infection, or in those at risk for MRSA or other gram-positive infection. The use and benefit of vancomycin should be balanced with the risk of potential renal toxicity. Fungal coverage should also be considered. Acyclovir should be strongly considered in all newborns with ALF until HSV PCR is negative (59).

SBP has been reported in 17-30\% of children with CLD and ascites, and index of suspicion must be high for its diagnosis $(60,61)$. While SBP can be diagnosed clinically, obtaining an ascitic fluid sample is necessary to document the presence of SBP (Table 4) with identification of a causative pathogen. The typical pathogens implicated in SBP include Streptococcus pneumoniae, Klebsiella, Escherichia coli, and Enterococcus. Therapy should consist of broadspectrum antibiotic therapy if the ascitic fluid analysis shows a polymorphonuclear leukocyte count of $\geq 250$ cells $/ \mathrm{mm}^{3}$. Amoxicillin-clavulanate, co-trimoxazole, and ciprofloxacin are commonly used for secondary prophylaxis against SBP $(30,59,60)$.

\section{Endocrine}

Adrenal insufficiency can occur in both ALF and ACLF, and is thought to be multifactorial in pathophysiology. It can be diagnosed with an ACTH stimulation test and treated with exogenous corticosteroid supplementation. Since cortisol is albumin-bound, low serum albumin levels may also account for low cortisol levels in these patients. Free cortisol detection is difficult and expensive, and is therefore not used in routine practice (62).

\section{Fluids, electrolytes, and nutrition}

Maintenance of intravascular volume is of the utmost importance in ALF. Volume status can be rapidly assessed using point-of-care cardiac ultrasound to determine whether to administer fluid for volume expansion. Hyponatremia and hypoglycemia may worsen neurologic injury from cerebral edema and increased ICP. Electrolyte abnormalities including hypophosphatemia, hypocalcemia, and hypomagnesemia are common and should be corrected. 
In ACLF, hydration status can be difficult to assess due to the presence of ascites and total body edema, but intravascular depletion is common. Lactate should not be used as a surrogate marker of intravascular fluid status, as it is metabolized by the liver and its clearance will be delayed. Hyponatremia is unavoidable in CLD and ACLF. Careful consideration should be made for any correction of sodium levels in ACLF due to risk for worsening of ascites as discussed below $(8,30)$.

Hyponatremia in ACLF can be quite profound, requiring ICU admission for careful partial correction and close monitoring. The mechanism is secondary to release of arginine vasopressin and anti-diuretic hormone, as well as renin-angiotensin axis activation leading to increased water intake that can potentiate the hyponatremia. Care must be taken to not correct hyponatremia too quickly, as this could lead to central pontine myelinolysis, quadriplegia, or coma. In addition, overcorrection may exacerbate ascites and fluid overload. It is important to note that blood products and albumin can inadvertently raise serum sodium too quickly. Hypokalemia is also common and should be corrected give that it may exacerbate HE by increasing renal ammonia production (30).

Many children with ACLF have concomitant moderate to severe malnutrition and it is an important determinant of post-transplant morbidity and mortality. Children with ALF are typically previously healthy and therefore do not usually have issues with malnutrition. Malnutrition occurs due to a combination of decreased energy intake, hypercatabolic state, and decreased nutrient absorption $(63,64)$. An aggressive approach to ensure adequate nutritional repletion, as well as correcting vitamin and micronutrient deficiencies is central to maintain remaining hepatic function, improve the patient's metabolic reserves, and the outcomes after liver transplantation (63-65).

Enteral nutrition (EN) with a gastric or jejunal smallbore feeding tube is the preferable route of delivery of nutrition for all patients who are not able to maintain adequate oral intake to maintain the integrity of the gastric mucosa and gut barrier. It is also less costly, with fewer complications and decreased hospital length of stay compared with parenteral nutrition $(\mathrm{PN})$ which carries risk of infection, fluid overload and electrolyte imbalance. Continuous nasogastric feeds are preferred over bolus feeds, to prevent fluctuations in glucose levels. Carbohydrate intake should exclusively be provided by glucose and cover $50-60 \%$ of non-protein energy requirements. Since fat is important in nutrient repletion of the malnourished patient, dietary fat should not be restricted unless true fat malabsorption has been diagnosed. Incorporating medium chain triglycerides, as an alternative form of fat not requiring bile salts for absorption, can provide a concentrated source of calories to patients with fat malabsorption. Unless a metabolic disease is suspected, protein content of diet should not be restricted, as this will worsen catabolism leading to more severe HE. Wholeprotein formulas are generally recommended, and branched chain amino-acid (BCAA) enriched formulas may be considered in patients who develop HE during re-feeding as BCAA are not metabolized in the liver. The registered dietitian (RD) plays an important role to assess nutrition therapy for both macro and micro-nutrients, including vitamins and minerals. Sodium may be added to enteral feeds to manage hyponatremia but close monitoring of sodium levels is warranted to limit overcorrection of sodium to prevent worsening of edema and ascites. Bowel regimens must be adjusted to optimize stooling in these children and avoid constipation (66).

\section{Extracorporeal liver support systems}

Over the past several decades, the efficacy of extracorporeal liver support systems has been evaluated as a bridge to liver transplantation or recovery. Much of these studies are focused on the adult population. Artificial liver support, including albumin dialysis devices, plasma exchange, and plasma pheresis are done at selected pediatric liver transplant centers worldwide. Bioartificial liver support, including hepatocyte transplantation, is still in its very early stages in pediatrics with very little data (67).

Albumin dialysis removes larger albumin-bound molecules that traditional hemodialysis cannot (including bilirubin, bile acids, amino acid metabolites, medium chain fatty acids, and cytokines). These albumin-bound molecules are thought to significantly contribute to the progression of ALF and ACLF, and the removal of these "toxins" is thought to slow this progression. Two commonly-used albumin dialysis devices are: Single Pass Albumin Dialysis (SPAD) and Molecular Adsorbents Recirculating System (MARS). The disadvantages of these systems are that they are expensive, not widely available, and there are limited data for survival benefit with these devices, particularly in the pediatric population (67).

Plasma exchange (PLEX) or plasmapheresis therapy removes plasma from the patient and replaces it with FFP or albumin. PLEX can be used in ALF as a bridge to 
transplantation to manage coagulation dysregulation (67). High flux continuous renal replacement therapy (CRRT) has also been used to remove ammonia and treat acute renal failure associated with ALF or ACLF (52). An advantage of these therapies is that they are typically available in an ICU environment. Many of these extracorporeal therapies can also be combined for maximum effect (e.g., SPAD with PLEX, CRRT with PLEX) (67).

\section{Conclusions}

The care of children with ALF and ACLF is complex and nuanced due to multiple organ systems that are affected with a very high risk for deterioration and death. The care of these patients in the ICU requires a multi-disciplinary approach with critical care physicians, hepatologists, transplant surgeons, nutritionists, pharmacists, social workers, and behavioral health personnel. Further study is needed to define research gaps in children with ACLF in particular, due to the lack of established criteria for in pediatrics, so these at-risk children can be identified early to optimize their readiness for liver transplantation.

\section{Acknowledgments}

Funding: None.

\section{Footnote}

Provenance and Peer Review: This article was commissioned by the editorial office, Translational Pediatrics for the series "Pediatric Critical Care". The article has undergone external peer review.

Peer Review File: Available at http://dx.doi.org/10.21037/tp20-375

Conflicts of Interest: All authors have completed the ICMJE uniform disclosure form (available at http://dx.doi. org/10.21037/tp-20-375). The series "Pediatric Critical Care" was commissioned by the editorial office without any funding or sponsorship. VS served as the unpaid Guest Editor of the series and serves as an unpaid editorial board member of Translational Pediatrics from Oct 2019 to Sep 2021. The other authors have no conflicts of interest to declare.

Ethical Statement: The authors are accountable for all aspects of the work in ensuring that questions related to the accuracy or integrity of any part of the work are appropriately investigated and resolved.

Open Access Statement: This is an Open Access article distributed in accordance with the Creative Commons Attribution-NonCommercial-NoDerivs 4.0 International License (CC BY-NC-ND 4.0), which permits the noncommercial replication and distribution of the article with the strict proviso that no changes or edits are made and the original work is properly cited (including links to both the formal publication through the relevant DOI and the license). See: https://creativecommons.org/licenses/by-nc-nd/4.0/.

\section{References}

1. American Liver Foundation. Pediatric Liver Research Agenda 2000: A Blueprint for the Future 2000.

2. Arya G, Balistreri WF. Pediatric liver disease in the United States: Epidemiology and impact. J Gastroenterol Hepatol 2002;17:521-5.

3. Squires RH, $\mathrm{Ng} \mathrm{V}$, Romero R, et al. Evaluation of the pediatric patient for liver transplantation: 2014 practice guideline by the AASLD, AST and NASPGHAN. Hepatology 2014;60:362-98.

4. Organ Procurement and Transplantation Network. OPTN Policies [Internet]. Policy 9: Allocation of Livers and Liver-Intestines 2020:163-216. Available online: https:// optn.transplant.hrsa.gov/media/1200/optn_policies.pdf

5. Squires RH, Shneider BL, Bucuvalas J, et al. Acute liver failure in children: The first 348 patients in the pediatric acute liver failure study group. J Pediatr 2006;148:652-8.

6. Devictor D, Desplanques L, Debray D, et al. Emergency liver transplantation for fulminant liver failure in infants and children. Hepatology 1992;16:1156-62.

7. Rivera-Penera T, Moreno J, Skaff C, et al. Delayed encephalopathy in fulminant hepatic failure in the pediatric population and the role of liver transplantation. J Pediatr Gastroenterol Nutr 1997;24:128-34.

8. Lutfi R, Abulebda K, Nitu ME, et al. Intensive Care Management of Pediatric Acute Liver Failure. J Pediatr Gastroenterol Nutr 2017;64:660-70.

9. Nel E, Sokol RJ, Comparcola D, et al. Viral Hepatitis in Children. J Pediatr Gastroenterol Nutr 2012;55:500-5.

10. Sundaram SS, Alonso EM, Narkewicz MR, et al. Characterization and outcomes of young infants with acute liver failure. J Pediatr 2011;159:813-818.e1.

11. Narkewicz MR, Olio DD, Karpen SJ, et al. Pattern of 
diagnostic evaluation for the causes of pediatric acute liver failure: An opportunity for quality improvement. J Pediatr 2009;155:801-6.e1.

12. Sarin SK, Choudhury A, Sharma MK, et al. Acute-onchronic liver failure: consensus recommendations of the Asian Pacific association for the study of the liver (APASL): an update. Hepatol Int 2019;13:353-90.

13. Moreau R, Jalan R, Gines P, et al. Acute-on-chronic liver failure is a distinct syndrome that develops in patients with acute decompensation of cirrhosis. Gastroenterology 2013;144:1426-37, 1437.e1-9.

14. O'Leary JG, Reddy KR, Garcia-Tsao G, et al. NACSELD Acute-on-Chronic Liver Failure (NACSELD-ACLF) Score Predicts 30-Day Survival in Hospitalized Patients with Cirrhosis. Hepatology 2018;67:2367-74.

15. Jagadisan B, Srivastava A, Yachha SK, et al. Acute on chronic liver disease in children from the developing world: Recognition and prognosis. J Pediatr Gastroenterol Nutr 2012;54:77-82.

16. D'Souza R, Grammatikopoulos T, Pradhan A, et al. Acute-on-chronic liver failure in children with biliary atresia awaiting liver transplantation. Pediatr Transplant 2019;23:e13339.

17. Alam S, Lal BB, Sood V, et al. Pediatric Acute-on-Chronic Liver Failure in a Specialized Liver Unit. J Pediatr Gastroenterol Nutr 2016;63:400-5.

18. Lal J, Thapa BR, Rawal P, et al. Predictors of outcome in acute-on-chronic liver failure in children. Hepatol Int 2011;5:693-7.

19. Zieve L. The mechanism of hepatic coma. Hepatology 1981;1:360-5.

20. Matsuura T, Yoshimaru K, Yanagi Y, et al. Massive pulmonary hemorrhage before living donor liver transplantation in infants. Pediatr Transplant 2016;20:89-95.

21. Deep A, Jose B, Dhawan A. Hepatopulmonary syndrome in children-an update. Paediatr Child Health (Oxford) 2015;25:282-5.

22. Krowka MJ, Fallon MB, Kawut SM, et al. International liver transplant society practice guidelines: Diagnosis and management of hepatopulmonary syndrome and portopulmonary hypertension. Transplantation 2016;100:1440-52.

23. Durand P, Baujard C, Grosse AL, et al. Reversal of hypoxemia by inhaled nitric oxide in children with severe hepatopulmonary syndrome, type 1 , during and after liver transplantation. Transplantation 1998;65:437-9.

24. Taniai N, Onda M, Tajiri T, et al. Reversal of hypoxemia by inhaled nitric oxide in a child with hepatopulmonary syndrome after living-related liver transplantation. In: Transplantation Proceedings. Elsevier; 2002:2791-2.

25. Schiller O, Avitzur Y, Kadmon G, et al. Nitric oxide for post-liver-transplantation hypoxemia in pediatric hepatopulmonary syndrome: Case report and review. Pediatr Transplant 2011;15:E130-4.

26. Chan-Dominy AC, Rahiman SN, Anders M, et al. Extracorporeal membrane oxygenation and severe portopulmonary hypertension following liver transplantation: Brief report. Int J Artif Organs 2015;38:337-42.

27. Dm T, Bt N, Ntn T, et al. Hepatic Hydrothorax-Rare Complication in Pediatrics. Int J Pediat Heal Care Adv 2015;2:10-2.

28. Sira MM, Sira MM, Behairy BES, et al. Hepatic hydrothorax in the absence of ascites in a child with autoimmune hepatitis: Successful management with octreotide and pleurodesis. Turk J Gastroenterol 2013;24:174-83.

29. Efrati O, Barak A. Pleural effusions in the pediatric population. Pediatr Rev 2002;23:417-26.

30. Shanmugam N, Dhawan A. Pediatric Liver Intensive Care. 2018:1-215.

31. Stravitz RT, Kramer AH, Davern T, et al. Intensive care of patients with acute liver failure: Recommendations of the U.S. Acute Liver Failure Study Group. Crit Care Med 2007;35:2498-508.

32. Harrison PM, Wendon JA, Gimson AES, et al. Improvement by Acetylcysteine of Hemodynamics and Oxygen Transport in Fulminant Hepatic Failure. N Engl J Med 1991;324:1852-7.

33. Lee WM, Hynan LS, Rossaro L, et al. Intravenous N-Acetylcysteine Improves Transplant-Free Survival in Early Stage Non-Acetaminophen Acute Liver Failure. Gastroenterology 2009;137:856-64, 864.e1.

34. Kortsalioudaki C, Taylor RM, Cheeseman P, et al. Safety and efficacy of $\mathrm{N}$-acetylcysteine in children with nonacetaminophen-induced acute liver failure. Liver Transpl 2008;14:25-30.

35. Squires RH, Dhawan A, Alonso E, et al. Intravenous $\mathrm{N}$-acetylcysteine in pediatric patients with nonacetaminophen acute liver failure: A placebocontrolled clinical trial. Hepatology 2013;57:1542-9.

36. Junge N, Junge C, Schröder J, et al. Pediatric cirrhotic cardiomyopathy: Impact on liver transplant outcomes. Liver Transpl 2018;24:820-30.

37. Gorgis NM, Kennedy C, Lam F, et al. Clinical 
Consequences of Cardiomyopathy in Children With Biliary Atresia Requiring Liver Transplantation. Hepatology 2019;69:1206-18.

38. $\mathrm{Ng} \mathrm{VL,} \mathrm{Li} \mathrm{R,} \mathrm{Loomes} \mathrm{KM,} \mathrm{et} \mathrm{al.} \mathrm{Outcomes} \mathrm{of} \mathrm{children}$ with and without hepatic encephalopathy from the pediatric acute liver failure study group. J Pediatr Gastroenterol Nutr 2016;63:357-64.

39. Blei AT, Larsen FS. Pathophysiology of cerebral edema in fulminant hepatic failure. J Hepatol 1999;31:771-6.

40. Tranah TH, Paolino A, Shawcross DL. Pathophysiological Mechanisms of Hepatic Encephalopathy. Clin Liver Dis (Hoboken) 2015;5:59-63.

41. Scott TR, Kronsten VT, Hughes RD, et al. Pathophysiology of cerebral oedema in acute liver failure. World J Gastroenterol 2013;19:9240-55.

42. Wright G, Sharifi Y, Jover-Cobos M, et al. The brain in acute on chronic liver failure. Metab Brain Dis 2014;29:965-73.

43. Bruno CM, Neri S, Sciacca C, et al. Plasma erythropoietin levels in anaemic and non-anaemic patients with chronic liver diseases. World J Gastroenterol 2004;10:1353-6.

44. Triantafyllou E, Woollard KJ, McPhail MJW, et al. The Role of Monocytes and Macrophages in Acute and Acute-on-Chronic Liver Failure. Front Immunol 2018;9:2948.

45. Mitchell O, Feldman DM, Diakow M, et al. The pathophysiology of thrombocytopenia in chronic liver disease. Hepat Med 2016;8:39-50.

46. Harrison MF. The misunderstood coagulopathy of liver disease: A review for the acute setting. West J Emerg Med 2018;19:863-71.

47. Raffini L, Witmer C. Pediatric transplantation: Managing bleeding. J Thromb Haemost 2015;13:S362-9.

48. Drebes A, de Vos M, Gill S, et al. Prothrombin Complex Concentrates for Coagulopathy in Liver Disease: SingleCenter, Clinical Experience in 105 Patients. Hepatol Commun 2019;3:hep4.1293.

49. Kodali S, Holmes CE, Tipirneni E, et al. Successful management of refractory bleeding in liver failure with tranexamic acid: Case report and literature review. Res Pract Thromb Haemost 2019;3:424-8.

50. Nair GB, Lajin M, Muslimani A. A Cirrhotic Patient With Spontaneous Intramuscular Hematoma Due to Primary Hyperfibrinolysis. Clin Adv Hematol Oncol 2011;9:249-52.

51. Gunawan B, Runyon B. The efficacy and safety of $\varepsilon$-aminocaproic acid treatment in patients with cirrhosis and hyperfibrinolysis. Aliment Pharmacol Ther 2006;23:115-20.

52. Deep A, Saxena R, Jose B. Acute kidney injury in children with chronic liver disease. Pediatr Nephrol 2019;34:45-59.

53. Moore JK, Love E, Craig DG, et al. Acute kidney injury in acute liver failure: A review. Expert Rev Gastroenterol Hepatol 2013;7:701-12.

54. Leventhal TM, Liu KD. What a Nephrologist Needs to Know About Acute Liver Failure. Adv Chronic Kidney Dis 2015;22:376-81.

55. Kulkarni S, Perez C, Pichardo C, et al. Use of Pediatric Health Information System database to study the trends in the incidence, management, etiology, and outcomes due to pediatric acute liver failure in the United States from 2008 to 2013. Pediatr Transplant 2015;19:888-95.

56. Grammatikopoulos T, Mckiernan PJ, Dhawan A. Portal hypertension and its management in children. Arch Dis Child 2018;103:186-91.

57. Shneider BL, Bosch J, De Franchis R, et al. Portal hypertension in children: Expert pediatric opinion on the report of the Baveno v consensus workshop on methodology of diagnosis and therapy in portal hypertension. Pediatr Transplant 2012;16:426-37.

58. Koul PB, Totapally BR, Raszynski A. Continuous octreotide infusion for treatment of upper gastrointestinal bleeding due to portal hypertension in children: An observational study from pediatric intensive care unit. J Pediatr Intensive Care 2012;1:99-103.

59. Bolia R, Srivastava A, Marak R, et al. Prevalence and Impact of Bacterial Infections in Children With Liver Disease-A Prospective Study. J Clin Exp Hepatol 2018;8:35-41.

60. Srivastava A, Malik R, Bolia R, et al. Prevalence, Clinical Profile, and Outcome of Ascitic Fluid Infection in Children With Liver Disease. J Pediatr Gastroenterol Nutr 2017;64:194-9.

61. El-Shabrawi MHF, El-Sisi O, Okasha S, et al. Diagnosis of spontaneous bacterial peritonitis in infants and children with chronic liver disease: A cohort study. Ital J Pediatr 2011;37:26.

62. Rakici H. Adrenal Insufficiency in Cirrhosis Patients: Evaluation of 108 Case Series. Euroasian J Hepatogastroenterol 2017;7:150-3.

63. Protheroe SM, Kelly DA. Cholestasis and end-stage liver disease. Baillieres Clin Gastroenterol 1998;12:823-41.

64. McCullough AJ. Malnutrition in liver disease. Liver Transpl 2000;6:S85-96.

65. Pierro A, Koletzko B, Carnielli V, et al. Resting energy 
expenditure is increased in infants and children with extrahepatic biliary atresia. J Pediatr Surg 1989;24:534-8.

66. Young S, Kwarta E, Azzam R, et al. Nutrition assessment and support in children with end-stage liver disease. Nutr
Clin Pract 2013;28:317-29.

67. Jain V, Dhawan A. Extracorporeal Liver Support Systems in Paediatric Liver Failure. J Pediatr Gastroenterol Nutr 2017;64:855-63.
Cite this article as: Mataya LA, Srinivasan V, Rand EB, Alcamo AM. Multiple organ involvement and ICU considerations for the care of acute liver failure (ALF) and acute on chronic liver failure (ACLF) in children. Transl Pediatr 2021;10(10):27492762. doi: $10.21037 /$ tp-20-375 